TESA sperm retrieval rates and ICSI outcomes in severe oligozoospermia

\title{
Sperm retrieval and intracytoplasmic sperm injection outcomes with testicular sperm aspiration in men with severe oligozoospermia and cryptozoospermia
}

\author{
Mohannad Alharbi, MD ${ }^{1,3}$; Ahmad Almarzouq, MD ${ }^{1}$; Armand Zini, MD ${ }^{1,2}$ \\ ${ }^{1}$ Division of Urology, Department of Surgery, McGill University, Montreal, QC, Canada; ${ }^{2}$ OVO Fertility Clinic, \\ Montreal, QC, Canada; ${ }^{3}$ Department of Surgery, College of Medicine, Qassim University, Qassim, Saudi Arabia
}

Cite as: Alharbi M, Almarzouq A, Zini A. Sperm retrieval and intracytoplasmic sperm injection outcomes with testicular sperm aspiration in men with severe oligozoospermia and cryptozoospermia. Can Urol Assoc J 2020 October 27; Epub ahead of print.

http://dx.doi.org/10.5489/cuaj.6798

Published online October 27, 2020

$* * *$

\section{Abstract}

Introduction: Several studies addressed the role of testicular sperm aspiration with intracytoplasmic sperm injection (ICSI) in azoospermic men but few have included nonazoospermic men. The aim of this study was to evaluate testicular sperm aspiration (TESA) sperm retrieval rates and ICSI outcomes in men with severe oligozoospermia.

Methods: Data were collected retrospectively from 88 consecutive, non-azoospermic, infertile men with idiopathic severe oligozoospermia who underwent TESA between January 2011 and January 2018. Patients were categorized into four groups according to sperm concentration: $<5$ and $>1 \mathrm{million} / \mathrm{ml}$ (group 1), <1 and $>0.1 \mathrm{million} / \mathrm{ml}$ (group 2), <0.1 million/ml (group 3) and cryptozoospermia (group 4).

Results: Mean male age was $37 \pm 7$ years and the mean female age was $33 \pm 4$ years. Sperm was recovered successfully in $90 \%(79 / 88)$ of the men overall and in $100 \%(30 / 30)$ of the men in group $1,97 \%(29 / 30)$ of the men in group $2,88 \%(15 / 17)$ of the men in group 3, and $45 \%$ $(5 / 11)$ of the men in group 4 . Most $(65 \%, 57 / 88)$ of the couples had an embryo transfer (ET). The overall clinical pregnancy rate per ET was $46 \%$ (26/57). The clinical pregnancy rates (per ET) were $43 \%(9 / 21)$ in group $1,65 \%(13 / 20)$ in group 2, 36\% (4/11) in group 3 and $0 \%(0 / 5)$ in group 4.

Conclusions: Our data indicate TESA allows for high sperm retrieval rates and acceptable ICSI pregnancy rates in men with severe oligozoospermia. However, in our experience, TESA sperm retrieval rates and ICSI outcomes are poor in cryptozoospermic men. 


\section{Introduction}

Several studies have evaluated the role of testicular sperm aspiration (TESA) with intracytoplasmic sperm injection (ICSI) in azoospermic men but few have examined TESA in nonazoospermic men. Since the introduction of intracytoplasmic sperm injection (ICSI) in the treatment of infertility, several controversies have arisen. Early reports illustrated that ICSI success is not influenced by sperm parameters such as concentration, morphology or motility and only injection of immotile sperm influenced ICSI outcomes negatively ${ }^{1-5}$ With more research conducted in this field, it was shown that severe asthenozoospermia can adversely impact pregnancy rates when compared to cases with higher than $5 \%$ sperm motility. ${ }^{6}$ Moreover, sperm morphology was similarly shown to negatively impact ICSI outcomes. ${ }^{7,8}$

There is mounting evidence to show that severe asthenozoospermia and cryptozoospermia can negatively affect fertilization and clinical pregnancy rates with ICSI. ${ }^{9}$ These findings may be explained by the high probability of sperm DNA damage and chromosomal abnormalities in men with low sperm counts..$^{10}$ Furthermore, sperm DNA damage was found to be a predictor of negative ICSI outcomes independent of concentration, morphology or motility. ${ }^{11-13}$ With these findings in mind, several researchers observed that ejaculated sperm is subject to more DNA damage than sperm acquired from the testis in both human and animal studies. ${ }^{14,15}$ These findings prompted further research in the utilization of testicular sperm in ICSI and several early reports showed superior pregnancy and live births but lower miscarriage rates with the use of testicular rather than ejaculated sperm-ICSI in couples with severe oligozoospermia (spermatozoa count $<5$ million / $\mathrm{ml}$ ) and cryptozoospermia (few sperm in the ejaculate that they are identified only after centrifugation of semen sample) $.9,16-18$ These findings were challenged with a meta-analysis that failed to show a difference in ICSI pregnancy rates between testicular and ejaculated sperm-ICSI in cryptozoospermic couples. ${ }^{19}$ Taking all the evidence into account, there is no robust evidence to show that severely oligozoospermic men have worse outcomes with ejaculate compared to testicular sperm-ICSI even in absence of high DNA fragmentation. However, the evidence that severe oligozoospermia and sperm DNA damage impact the success rate of ICSI when ejaculated sperm are used remains. ${ }^{9}$ In this study we sought to evaluate TESA sperm retrieval rates and ICSI outcomes in men with severe oligozoospermia and cryptozoospermia.

\section{Methods}

\section{Patients}

Data were collected retrospectively from 88 consecutive, non-azoospermic infertile men with idiopathic severe oligozoospermia (spermatozoa count $<5$ million $/ \mathrm{ml}$ ) or cryptozoospermia (few sperm in the ejaculate that they are identified only after centrifugation of semen sample) who underwent TESA after one or more failed ICSI attempt(s) using ejaculated sperm between 
January 2011 and January 2018 at the OVO fertility clinic in Montreal, Canada. Patients were categorized into 4 groups according to sperm concentration: $<5$ and $>1 \mathrm{million} / \mathrm{ml}$ (group 1), $<1$ and $>0.1 \mathrm{million} / \mathrm{ml}$ (group 2), $<0.1 \mathrm{million} / \mathrm{ml}$ (group 3) and cryptozoospermia (group 4). We excluded couples with advanced female age ( $>40$ years). We also excluded couples with a correctable male factor (e.g. varicocele, semen infection).

Consent was not obtained from patients. The research and development scientific committee at OVO clinic reviewed our study and we acquired the approval as a quality control study. Also, we followed the Helsinki Declaration principle.

Semen analysis was done using a microptic SCA (Sperm Class Analyzer, Microptic, Barcelona, Spain) with measurements sperm motility taken at $37^{\circ} \mathrm{C}$. All men were evaluated in our clinic with a thorough history, physical examination and relevant laboratory testing. At our IVF center, TESA (testicular sperm aspiration) is done fresh the day before oocyte retrieval in keeping with our embryologist's preference. Before performing TESA-ICSI, every case was first reviewed by the clinical team (Urologist, Gynecologist, and Embryologist). The nature of testicular sperm-ICSI was discussed with the patients. Moreover, we informed the couples about the potential benefits and risks of testicular sperm retrieval (bleeding, infection, pain, hypogonadism, unknown genetic and epigenetic risks). In regard to patients with cryptozoospermia included in our study, they were counselled for microdissection testicular sperm extraction (microTESE) but they elected to undergo TESA. Genetic testing (karyotype \& Y-chromosome microdeletion) was done to all patients.

We collected the following variables in our study: patient and partner age, testicular volumes, serum follicle stimulating hormone (FSH) level, total testosterone, total number of embryos transferred (ET), sperm retrieval rates, and clinical pregnancy rate (per embryo transfer). Live birth rate was not assessed because of loss of follow up of most couples. We utilized the SCSA (sperm chromatin structure assay) to detect sperm DNA fragmentation $(\mathrm{SDF})$ and treated men with moderately high SDF $(>15.0 \%$ and $<30 \%)$ or high SDF $(>30.0 \%)$.

\section{Testicular sperm retrieval}

Testicular sperm retrieval was performed by testicular sperm aspiration (TESA) under local anesthesia and all procedures were performed by the same surgeon (AZ), as previously described.$^{20}$

\section{Statistical analysis}

IBM Statistical Package for the Social Sciences (SPSS, version 20; SPSS Inc., IBM Corp., Armonk, NY, USA) was used to collect data and perform statistical analysis. Continuous variables were expressed as mean $\pm \mathrm{SD}$ and were assessed using one-way ANOVA. Fisher's exact test was used to compare dichotomous variables. A p value $\leq 0.05$ was considered statistically significant. 


\section{Results}

We identified 88 patients that underwent testicular sperm ICSI. Although no early complications were reported, we could not adequately assess late TESA complications (e.g. chronic testicular pain, hypogonadism) because the majority of the patients did not return for follow up after TESA. The baseline characteristics, sperm retrieval rates and clinical pregnancy outcomes are shown in Table 1 . The mean $( \pm$ SD) male age was $37 \pm 7$ years and the mean female age was $33 \pm 4$ years. There was no significant difference in maternal or paternal age between the groups. The mean testicular volumes, serum FSH level, and number of embryos transferred were comparable among the four groups with no significant difference. We identified two men with genetic abnormalities: one with a 47 XYY- 45 X karyotype and another patient with a translocation (8:18). Bilateral TESA was done in two patients with the remainder undergoing unilateral TESA. Sperm was recovered successfully in $90 \%(79 / 88)$ of the men overall and in $100 \%(30 / 30)$ of the men in group 1 ( $>1$ to 5 million/ml), 97\% (29/30) of the men in group $2(0.1$ to $1 \mathrm{million} / \mathrm{ml}), 88 \%(15 / 17)$ of the men in group $3(<0.1$ million $/ \mathrm{ml})$ and $45 \%(5 / 11)$ of the men in group 4 (cryptoozospermia). $65 \%(57 / 88)$ of the couples had an embryo transfer. The overall clinical pregnancy rate per embryo transfer (ET) was $46 \%(26 / 57)$ with a mean of $1.8 \pm 0.3$ embryos transferred per cycle. The clinical pregnancy rates (per ET) were 43\% (9/21) in group 1, 65\% (13/20) in group 2, 36\% (4/11) in group 3 and $0 \%(0 / 5)$ in group 4 .

There was a statistically significant difference in sperm retrieval rates between the groups $(P<0.05)$ with less than $50 \%$ chance of finding sperm in men with cryptozoospermia. On the other hand, all men with a sperm concentration $>1 \mathrm{million} / \mathrm{ml}$ had a successful sperm retrieval. Furthermore, we observed a lower pregnancy outcome in couples with cryptozoospermia $(P=0.05)$.

\section{Discussion}

We have performed a retrospective study on sperm retrieval outcomes, and, clinical pregnancy rates in couples with severe oligozoospermia and cryptozoospermia undergoing TESA with ICSI. We have shown that, overall, TESA is associated with high sperm retrieval rates and acceptable ICSI pregnancy outcomes in men with severe oligozoospermia. However, in the subset of men with cryptozoosperma, TESA sperm retrieval rates and ICSI pregnancy rates were poor.

In our study, $90 \%$ of the men had successful sperm retrieval. Subgroup analysis showed that sperm were successfully recovered in $100 \%$ of the men in group 1 ( $>1$ to 5 million/ml), $97 \%$ of the men in group $2(0.1$ to $1 \mathrm{million} / \mathrm{ml}), 88 \%$ of the men in group $3(<0.1 \mathrm{million} / \mathrm{ml})$ and $45 \%$ of the men in group 4 (cryptoozospermia). The poor sperm retrieval rate in the cryptozoospermic men in our study (45\%) is comparable to that found by Alrabeeah et al. $(43 \%) .{ }^{21}$ With the exception of sperm concentration, there was no significant difference among 
the subgroups in term of baseline characteristics (age of couples, testicular volume, serum FSH level, number of ET) indicating a reasonable homogeneity in this cohort. Regarding the specific result of the two men with genetic abnormalities, both had successful sperm retrieval that resulted in pregnancy. The characteristic of the patient with a $47 \mathrm{XYY}-45 \mathrm{X}$ karyotype were $(<$ 1 million $/ \mathrm{ml}$ on semen analysis, $16 \mathrm{cc}$ testicular volume, $\mathrm{FSH}=4 \mathrm{IU} / \mathrm{L}$, total testosterone $=10$ $\mathrm{nmol} / \mathrm{L}, 1$ embryo transfer). The other patient with a translocation $(8: 18)$ had $(<5$ million $/ \mathrm{ml}$ on semen analysis, $20 \mathrm{cc}$ testicular volume, 2 embryos transfer).

The reason for using testicular sperm varied according to the subgroup. The men in group 1 had prior ICSI failure with evidence of high sperm DNA fragmentation on DNA testing. The men in groups 2,3 and 4 had failed 1 or more ICSI attempts using ejaculated sperm and were presumed to have sperm DNA fragmentation based on the association between sperm DNA fragmentation and standard sperm parameters (this could not be tested because the sperm DNA assay requires a minimum of 1 million cells $/ \mathrm{ml}){ }^{22}$

The use of testicular sperm in the context of severe oligozoospermia is based on the rationale that (1) sperm DNA fragmentation is common in men with severe oligozoospermia, (2) the level of sperm DNA fragmentation is significantly lower in testicular compared to ejaculated sperm and that (3) higher pregnancy rates are reported with the use of testicular compared to ejaculated sperm in men with high levels of sperm DNA fragmentation. ${ }^{14,16,23,24}$ Similarly, experimental studies have shown that in animals with abnormal spermatogenesis, the passage of sperm with poor chromatin compaction through the epididymis results in sperm DNA fragmentation and impaired fertility potential. ${ }^{15}$

We have found difference (albeit non-significant) in pregnancy rates per ET among the subgroups. The overall pregnancy rate in couples with severe oligozoospermia (groups 1,2 and 3 ) was $46 \%$. In contrast, no pregnancies were observed in the couples with cryptozoospermia. Although, there is paucity of data in the literature on pregnancy outcomes with TESA-ICSI for men with severe oligozoospermia and cryptozoospermia, the available studies demonstrate favourable pregnancy rates in such patients using testicular sperm harvested by microTESE. ${ }^{16,17,21}$ Mehta et al. reported a pregnancy rate of $50 \%$ in men with severe oligozoospermia and high DNA fragmentation index (DFI) using testicular sperm retrieved by microdissection testicular sperm extraction (micro-TESE) ${ }^{17}$ Moreover, Inal et al. evaluated men with severe oligozoospermia who underwent micro-TESE and found a $36 \%$ clinical pregnancy rate. ${ }^{25}$

The men in our study were stratified based on their sperm concentration because it has been shown that the degree of severity of oligozoospermia may negatively impact ICSI outcomes. ${ }^{9}$ The studies on cryptozoospermic men have shown conflicting results. Several studies have demonstrated that using testicular sperm is associated with higher pregnancy and live birth rates compared to ejaculated sperm in cryptozoospermic men. ${ }^{26-29}$ These observations support the belief that testicular sperm has better quality than ejaculated sperm through two 
explanations. First, the acquired sperm DNA damage during epidydimal transit. Second, semen samples in men with cryptozoospermia undergoes extensive processing to be used in ICSI which can increase oxidative stress and results in poor sperm quality which in turn has negative impact on ICSI outcomes. ${ }^{19}$ Conversely, other investigators did not show any differences in the ICSI outcomes using testicular versus ejaculated sperm in men with cryptozoospermia. ${ }^{19,30}$ Our data do not support the use of testicular sperm recovered by TESA in men with cryptoozospermia. We suspect that the poor outcomes in this study may be related to the retrieval method (TESA) as we have previously shown good sperm retrieval and pregnancy outcomes in these couples when microTESE-ICSI is performed. ${ }^{21}$

\section{Conclusions}

In conclusion, our study indicates that TESA allows for high sperm retrieval rates and acceptable ICSI pregnancy rates in men with severe oligozoospermia. However, in our experience, TESA sperm retrieval rates were poor in cryptozoospermic men suggesting that this sperm retrieval method is not optimal for this cohort. Moreover, ICSI outcomes were also poor in couples with cryptozoospermia. Certainly, there is a need for larger, well-designed, randomized, prospective studies to assess the value of sperm DNA fragmentation index and sperm retrieval techniques in men with severe oligozoospermia and cryptozoospermia. Moreover, such studies will determine which subgroups may benefit most from use of testicular sperm in ICSI. 
TESA sperm retrieval rates and ICSI outcomes in severe oligozoospermia

\section{References}

1. Palermo G, Joris H, Devroey P \& Van Steirteghem AC. (1992) Pregnancies after intracytoplasmic injection of single spermatozoon into an oocyte. Lancet 340, 17-18.

2. Palermo G, Joris H, Derde MP, Camus M, Devroey P \& Van Steirteghem A. (1993) Sperm characteristics and outcome of human assisted fertilization by subzonal insemination and intracytoplasmic sperm injection. Fertil Steril 59, 826-835.

3. Nagy ZP, Liu J, Joris H, Verheyen G, Tournaye H, Camus M, Derde MC, Devroey P, Van Steirteghem AC. The result of intracytoplasmic sperm injection is not related to any of the three basic sperm parameters. Hum Reprod 1995;10:1123-1129.

4. Nijs M, Vanderzwalmen P, Vandamme B, Segal-Bertin G, Lejeune B, Segal L, van Roosendaal E, Schoysman R. Fertilizing ability of immotile sperma- tozoa after intracytoplasmic sperm injection. Hum Reprod 1996;11: 2180-2185.

5. Vandervorst M, Tournaye H, Camus M, Nagy ZP, Van Steirteghem A, Devroey P. Patients with absolutely immotile spermatozoa and intracy- toplasmic sperm injection. Hum Reprod 1997;12:2429-2433.

6. Mitchell V, Rives N, Albert M, Peers MC, Selva J, Clavier B, Escudier E, Escalier D. Outcome of ICSI with ejaculated spermatozoa in a series of men with distinct ultrastructural flagellar abnormalities. Hum Reprod 2006;21:2065-2074.

7. De Vos A, Van De Velde H, Joris H, Verheyen G, Devroey P, Van Steirteghem A. Influence of individual sperm morphology on fertilization, embryo morphology, and pregnancy outcome of intracytoplasmic sperm injection. Fertil Steril 2003;79:42-48.

8. Van den Hoven L, Hendriks JC, Verbeet JG, Westphal JR, Wetzels AM. Status of sperm morphology assessment: an evaluation of methodology and clinical value. Fertil Steril 2015; 103:53-58.

9. Strassburger D, Friedler S, Raziel A, Schachter M, Kasterstein E, Ron-el R. Very low sperm count affects the result of intracytoplasmic sperm injec- tion. J Assist Reprod Genet 2000;17:431-436.

10. De Braekeleer M, Dao TN. Cytogenetic studies in male infertility: a review. Hum Reprod 1991;6:245-250.

11. Zini A, Boman JM, Belzile E, Ciampi A. Sperm DNA damage is associated with an increased risk of pregnancy loss after IVF and ICSI: systematic review and metaanalysis. Hum Reprod 2008;23:2663-2668.

12. Zhao J, Zhang Q, Wang Y, Li Y. Whether sperm deoxyribonucleic acid fragmentation has an effect on pregnancy and miscarriage after in vitro fertilization/intracytoplasmic sperm injection: a systematic review and meta-analysis. Fertil Steril 2014;102:9981005 , e1008.

13. Oleszczuk K, Giwercman A, Bungum M. Sperm chromatin structure assay in prediction of in vitro fertilization outcome. Andrology 2016;4:290-296.

14. Greco E, Scarselli F, Iacobelli M, Rienzi L, Ubaldi F, Ferrero S, Franco G, Anniballo $\mathrm{N}$, Mendoza C, Tesarik J. Efficient treatment of infertility due to sperm DNA damage by ICSI with testicular spermatozoa. Hum Reprod 2005;20:226-230. 
15. Suganuma R, Yanagimachi R, Meistrich ML. Decline in fertility of mouse sperm with abnormal chromatin during epididymal passage as revealed by ICSI. Hum Reprod 2005;20:3101-3108.

16. Esteves SC, Sanchez-Martin F, Sanchez-Martin P, Schneider DT, Gosalvez J. Comparison of reproductive outcome in oligozoospermic men with high sperm DNA fragmentation undergoing intracytoplasmic sperm injection with ejaculated and testicular sperm. Fertil Steril 2015;104: 1398-1405.

17. Mehta A, Bolyakov A, Schlegel PN, Paduch DA. Higher pregnancy rates using testicular sperm in men with severe oligospermia. Fertil Steril 2015; 104:1382-1387.

18. Negri L, Patrizio P, Albani E, Morenghi E, Benaglia R, Desgro M, Levi Setti PE. ICSI outcome is significantly better with testicular spermatozoa in patients with necrozoospermia: a retrospective study. Gynecol Endocrinol 2014;30: 48-52.

19. Abhyankar N, Kathrins M, Niederberger C. Use of testicular versus ejaculated sperm for intracytoplasmic sperm injection among men with cryptozoospermia: a metaanalysis. Fertil Steril 2016;105:1469-1475.

20. Al-Malki A. H., K. Alrabeeah, E. Mondou, V. Brochu-Lafontaine, S. Phillips and A. Zini. (2017) Testicular sperm aspiration (TESA) for infertile couples with severe or complete asthenozoospermia. Andrology 5: 226-231

21. Alrabeeah K, Wachter A, Phillips S, Cohen B, Al-Hathal N, et al. Sperm retrieval outcomes with microdissection testicular sperm extraction (micro-TESE) in men with cryptozoospermia. Andrology 2015; 3: 462-6.

22. Moskovtsev, S.; Willis, J.; White, J.; Mullen, J. Sperm DNA Damage: Correlation to Severity of Semen Abnormalities. Urology 2009, 74 (4), 789-793.

23. Esteves SC, Roque M, Bradley CK, Garrido N. Reproductive outcomes of testicular versus ejaculated sperm for intracytoplasmic sperm injection among men with high levels of DNA fragmentation in semen: systematic review and meta-analysis. Fertil Steril 2017; 108: 456-67.e1.

24. Herrero MB, Lusignan MF, Son WY, Sabbah M, Buckett W, Chan P. ICSI outcomes using testicular spermatozoa in non-azoospermic couples with recurrent ICSI failure and no previous live births. Andrology. 2019 May;7(3):281-287.

25. Inal HA, Inci Kahyaoglu, Ayten Turkkani, Deniz Tuzluoglu, Nafiye Yilmaz. Retrospective comparison of intracytoplasmic sperm injection outcomes of sperm retrieved from a testicular biopsy and freshly ejaculated semen in oligozoospermia. Rev Int Androl. 2018 Oct - Dec;16(4):131-136.

26. Ben-Ami I, Raziel A, Strassburger D, Komarovsky D, Ron-El R \& Friedler S. (2013) Intracytoplasmic sperm injection outcome of ejaculated versus extracted testicular spermatozoa in cryptozoospermic men. Fertil Steril 99, 1867-1871.

27. Bendikson KA, Neri QV, Takeuchi T, Toschi M, Schlegel PN, Rosenwaks Z \& Palermo GD. (2008) The outcome of intracytoplasmic sperm injection using occasional spermatozoa in the ejaculate of men with spermatogenic failure. J Urol 180, 1060-1064.

28. Hauser R, Bibi G, Yogev L, Carmon A, Azem F, Botchan A, Yavetz H, Klieman SE, Lehavi O, Amit A \& Ben-Yosef D. (2011) Virtual azoospermia and cryptozoospermia - 
fresh/frozen testicular or ejaculate sperm for better IVF outcome? J Androl 32, 484490.

29. Cui X, Ding P, Gao G \& Zhang Y (2016) Comparison of the clinical outcomes of intracytoplasmic sperm injection between spermatozoa retrieved from testicular biopsy and from ejaculate in cryptozoospermia patients. Urology 102, 106-110.

30. Amirjannati N, Heidari-Vala H, Akhondi MA, Hosseini Jadda SH, Kamali K \& Sadeghi MR. (2012) Comparison of intracytoplasmic sperm injection outcomes between spermatozoa retrieved from testicular biopsy and from ejaculation in cryptozoospermic men. Andrologia 44 (Suppl. 1), 704-709. 
Figures and Tables

\begin{tabular}{|l|c|c|c|c|c|}
\hline \multicolumn{6}{|l|}{$\begin{array}{l}\text { Table 1. Clinical characteristics, sperm retrieval outcomes and clinical pregnancy rates in couples } \\
\text { with severe oligozoospermia and cryptozoospermia managed by TESA with ICSI }\end{array}$} \\
\hline & Cryptozoospermia & $<\mathbf{0 . 1} \mathbf{M}$ & $\mathbf{0 . 1}-\mathbf{1} \mathbf{M}$ & $\mathbf{1 - 5} \mathbf{M}$ & $\mathbf{p}$ \\
\hline $\mathrm{n}$ & 11 & 17 & 30 & 30 & \\
\hline Male age & $34.6 \pm 1.5$ & $37.9 \pm 1.5$ & $35 \pm 1$ & $39.1 \pm 1.4$ & $0.052^{\mathrm{a}}$ \\
\hline Female age & $33.5 \pm 1.1$ & $33 \pm 9.9$ & $32 \pm 0.8$ & $33.6 \pm 0.73$ & $0.47^{\mathrm{a}}$ \\
\hline Right testicular volume (mL) & $16.1 \pm 0.9$ & $15.1 \pm 0.9$ & $14.7 \pm 0.8$ & $16 \pm 0.7$ & $0.55^{\mathrm{a}}$ \\
\hline Left testicular volume (mL) & $15.5 \pm 0.9$ & $14.4 \pm 0.7$ & $14.6 \pm 0.7$ & $15.4 \pm 0.8$ & $0.73^{\mathrm{a}}$ \\
\hline FSH (IU/L) & $12.4 \pm 3.3$ & $10.3 \pm 1.7$ & $10.2 \pm 1.9$ & $11.1 \pm 1.7$ & $0.91^{\mathrm{a}}$ \\
\hline Total testosterone (nmol/L) & $14.5 \pm 6.1$ & $15.5 \pm 2.1$ & $11.1 \pm 5.1$ & $11.8 \pm 1.1$ & $0.50^{\mathrm{a}}$ \\
\hline Number of embryos transferred & $2 \pm 0.32$ & $1.7 \pm 0.24$ & $1.95 \pm 0.3$ & $1.7 \pm 0.16$ & $0.73^{\mathrm{a}}$ \\
\hline Successful sperm retrievals (\%) & $5 / 11(45)$ & $15 / 17(88)$ & $29 / 30(97)$ & $30 / 30(100)$ & $<0.001^{\mathrm{b}}$ \\
\hline Pregnancy rate per ET (\%) & $0 / 5(0)$ & $4 / 11(36)$ & $13 / 20(65)$ & $9 / 21(43)$ & $0.05^{\mathrm{b}}$ \\
\hline
\end{tabular}

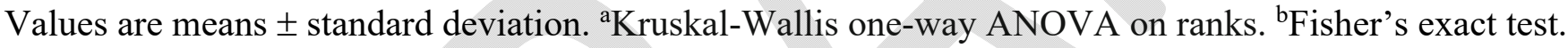
ET: embryo transfer; FSH: follicle-stimulating hormone; ICSI: intra-cytoplasmic sperm injection; TESA: testicular sperm aspiration. 